\title{
Should Herbs Take All the Blame? Causality Assessment of a Serious Thrombocytopenia Event
}

\author{
Jung-Nien Lai, MD, PhD, ${ }^{1-3}$ Shu-Ching Hsieh, RN, PhD, ${ }^{4}$ Pau-Chung Chen, MD, PhD, ${ }^{5}$ \\ Huey-Jen Chen, MD, and Jung-Der Wang, MD, ScD ${ }^{5,7}$
}

\section{Abstract}

Background: With the increasing use of herbal medicines, the causality assessment of adverse drug-related reactions becomes more complicated because of the concomitant use of herbs and conventional medications. Epidemiological causal inference can be a central feature of such judgment but may be insufficient. Other scientific considerations include study design, bias, confounding, and measurement issues. The approach of this study is to establish an active safety surveillance system for finished herbal products (FHPs) and to review each adverse event regularly.

Method: A single case of serious thrombocytopenia was found in 136 subjects taking FHPs on a clinical trial for 12 weeks, for which the cause was sought.

Results: Because at the end of the first month the patient's platelet counts were normal and the thrombocytopenia developed after the co-medication with conventional drugs, it was suspected that the thrombocytopenia might not be attributed to the use of FHP.

Conclusions: This report summarizes the criteria of causality assessment under mixed use of herbs and conventional medicine and recommends a feasible process for careful evaluation of adverse drug reactions related to all herbal medicine.

\section{Introduction}

$\mathbf{T}$ HE OCCURRENCE OF ADVERSE DRUG-RELATED events (ADRs) among herbal users has been progressively documented and reviewed. ${ }^{1-6}$ When both conventional and herbal medicines are available to lay people, there seems a tendency for them to be used concomitantly. ${ }^{7,8}$ Thus, based upon the current understanding of each single herb, along with their known interactions with conventional types of medication, it would seem inappropriate to infer the net effects of a mixed herbal regimen. ${ }^{9}$ Public health professionals, therefore, do not have the luxury of waiting for progress; rather, public health professionals involved in herbal medicine have a moral obligation to consider the need for herbal safety recommendations, given the available evidence.

Herbal safety recommendations cannot easily be separated from causal conclusions, which are a form of scientific statement that emerges from the application of criteria-based inferential methods. At a minimum, the set of criteria includes temporality, consistency, strength of association, dose response, and plausibility. The current practice, methods, and theory of causal inference in this case report permit flexibility in the choice of criteria, their relative priority, and the rules of inference assigned to them, which mostly follow the World Health Organization global introspection method. ${ }^{10}$

Finished herbal products (FHPs) were concentrated herbal extracts manufactured from one or more herbs into granular forms by pharmaceutical companies. Such a dosage form of FHPs is generally very convenient for patients in Korea, China, Japan, and Taiwan as traditional medicine, and for consumers in the United States as health foods or over the counter drugs. As a supplement to the current pharmacovigilance methods for the use of FHPs or for compounds of these products used in traditional Chinese herbal medicine (TCHM), we established an active safety surveillance system

\footnotetext{
${ }^{1}$ Departments of Obstetrics and Gynecology and ${ }^{2}$ Department of Chinese Medicine, Taipei City Hospital, Yangming Branch, Taipei, Taiwan.

${ }^{3}$ Institute of Traditional Medicine, School of Medicine, National Yangming University, Taipei, Taiwan.

${ }^{4}$ Center for Drug Evaluation, Taipei, Taiwan.

${ }^{5}$ Institute of Occupational Medicine and Industrial Hygiene, National Taiwan University College of Public Health, Taipei, Taiwan.

${ }^{6}$ Integrated Division of Menopause, Taipei Municipal Chinese Medical Hospital, Taipei, Taiwan.

${ }^{7}$ Department of Public Health, National Cheng Kung University, Tainan, Taiwan.
} 
that followed the principles of good clinical practice, and then carried out periodic evaluation of the patients for signs of any adverse events. To test the feasibility of the system, we first applied it to the evaluation of the safety and efficacy, in women in the climacteric, of a combination of three TCHM formulae, referred to as "TMN-1" and comprising 21 constituents (Table 1). After gaining the formal approval of the Joint Institutional Review Board, this study was carried out at four sites, where 136 subjects were recruited to take the medication; follow-up of the study subjects was then undertaken for a period of 12 weeks. $^{11}$

During the study period, there was an isolated occurrence of a serious adverse event (SAE)—acute thrombocytopenia. This article describes how causal criteria are used to help meet the challenge of making herbal safety recommendations in the face of complex scientific evidence.

\section{Case Report}

A 52-year-old woman, whose last menstrual period was in March 2004, was recruited into this study on June 6, 2004. She was afflicted with perimenopausal symptoms, including hot flashes, sweating, and irregular menstrual cycle, and had been seeking alternative medication to improve her symptoms. She had been taking TMN-1 3 times a day without any complaints until she discovered gingival bleeding (on the 55th day), which was instantly reported to the study nurse. The patient was required to return immediately to the study site to undergo a careful and thorough examination.

The investigator at the study site found that the woman had numerous petechiae and ecchymoses over her bilateral shoulders, forearms, palms, and thighs. Her complete blood count showed white blood cell count $3700 / \mathrm{mm}^{3}$ red blood cell count 4,150,000/mm, hemoglobin $12.6 \mathrm{mg} / \mathrm{dL}$, and platelets $2000 / \mathrm{mm}^{3}$ Her vital signs were within the normal range and she had no palpable hepatosplenomegaly or lymphadenopathy. The patient was immediately referred to a nearby medical center where her low platelet count (3000/ $\mathrm{mm}^{3}$ ) was verified. Given that her platelet count on the 28th day after medication had been $254,000 / \mathrm{mm}^{3}$ a diagnosis of acute thrombocytopenia was made and she immediately received 12 units of platelet transfusion.

Since her platelet count had returned to $99,000 / \mathrm{mm}^{3}$ by the following day, following consultation with a hematologist, she was subsequently discharged. As a result of the occurrence of this SAE, the patient was instructed to stop taking all medications (prescribed or otherwise) and dietary supplements. In her two subsequent follow-up visits, 1 week and 1 month after the SAE, she showed consistently normal platelet counts. The question that therefore arises from this SAE was whether the FHP should take all the blame, or whether some of the blame should be apportioned to TMN-1. During the process of looking for all possible causative agents, we found that there were concomitant uses of conventional medicines and other folk herbs before the occurrence of this SAE. We therefore pursued further details.

\section{Assessment of the Causality of the SAE}

Based upon ethical considerations, we did not rechallenge the patient with TMN-1, nor did we conduct any laboratory studies with any of the suspected drugs (including drugdrug or drug-herb combinations).

Despite Hill's criteria having first been proposed in the 1960s, different authors have proposed and emphasized different criteria of causality inference. ${ }^{12-15}$ From a refutational point of view, proposing necessary criteria can reduce the difficulty of determining causality, as such criteria will allow one to rule out other possible causal relationships. We proposed to prioritize causal criteria for herbal users, as follows:

\section{The Only Necessary Causal Criteria: Temporality}

Recently published epidemiology textbooks ${ }^{16,17}$ seem to regard "temporality" as the only necessary criterion, with all others being generally regarded as incrementally adding credibility to the inference of causality.

The SAE (platelet levels $3000 / \mathrm{mm}^{3}$ ) occurred in this patient after she had been taking TMN-1 for a period of 54 days (having consumed 147 sachets). Prior to the event, her platelet counts had been checked both before taking the drugs and at the 28th day of the trial: Her platelet levels were $241,000 / \mathrm{mm}^{3}$ at the outset, and $254,000 / \mathrm{mm}^{3}$ at the 4 -week point (when she had consumed 58 sachets). By definition of temporality, TMN-1 taking should precede this SAE, and a universal law of nature should remain in other participants, other clinical settings, and other time during the study period. To apply the criteria in this case, we must also take the minimal induction time and maximal latency period for TMN-1 to produce acute thrombocytopenia into consideration. For most participants who completed the trial, they had consumed TMN-1 with higher dose level and had exposed to the study drug with longer time period than the

Table 1. Constituents of TMN-1

Angelica sinensis (Oliv.) Diels (dong quai)

Atractylodes macrocephala Koidez. (bai zhu)

Paeonia lactiflora Pall. (garden peony)

Bupleurum chinense DC. (chai hu)

Poria cocos (Schw.) Wolf (poria)

Glycyrrhiza uralensis Fisch. (licorice root)

Paeonia suffruticosa Andr. (tree peony)

Gardenia jasminoides Ellis (gardenia)

Zingiber officinale Rosc. (ginger)

Mentha haplocalyx Briq. (corn mint)

Aucklandia lappa Decne. (yunmuxiang)
Rehmannia glutinosa Libosch. (Chinese foxglove)

Cornus officinalis Sieb.et Zucc. (Japanese cornel)

Dioscorea opposita Thunb. (Chinese yam)

Alisma orientalis Juzep. (water plantain)

Anemarrhena asphodeloides Bge. (zhi mu)

Phellodendron chinense Schneid (Chinese corktree)

Panax ginseng C. A. Meyer (ginseng)

Citrus reticulata Blanco (tangerine)

Pinellia ternata (Thunb.) Breit. (ban xia)

Amomum villosum Lour. (cardamom seed)

TMN-1, combination of three traditional Chinese herbal medicine formulae. 
SAE women. In addition, because the patient's platelet count remained almost the same level both before entering the trial and after taking 4 weeks of TMN-1. We could exclude the possibility for its long latency the thrombocytopenia is already present and processing at the time the SAE occurred after taking 4-week TMN-1. It therefore seemed extremely unlikely that the TMN-1 alone could have induced such acute thrombocytopenia in the patient.

Traditionally, one might apply the induction method to propose that TMN-1 was the cause of acute thrombocytopenia after observing this event. It is also known that one cannot easily prove a causality such as A causes B. However, a hypothesis can be refuted or proven to be false. For this case, the search for truth and causal mechanism is not end; the process of conjecture and refutation should continue indefinitely. However, the temporal conclusion can still be drawn that the study drug would be unlikely to induce acute thrombocytopenia alone.

\section{Causal Criteria: Consistency of Association}

The principle of consistency stipulated that a natural law guarantees that everyone will share a similar response profile from exposure to a specific toxic substance. A causal relationship can be found consistently under different times, participants, and settings. After careful review, among the 21 constituents of TMN-1 (Table 1), Angelica sinensis (dong quai) was once reported to have interacted with warfarin to cause prolonged thrombin time ${ }^{18}$; however, since none of the remaining constituents in TMN-1 has ever been reported to have any association with thrombocytopenia, the likelihood of the delayed effect described above would be very low. In addition, all participants were monitored carefully throughout the period of this trial. There was 1 participant who had an initial abnormal platelet count; however, this was attributed to her pre-existing diagnosis of von Willebrand disease, type I. This patient's platelet levels did not show any significant reduction during the study period, which might have been attributable to TMN-1. Her platelet levels were $114,000 / \mathrm{mm}^{3}$ at baseline, $64,000 / \mathrm{mm}^{3} 4$ weeks after first taking TMN-1, and $82,000 / \mathrm{mm}^{3}$ at the 12 -week point of the trial. Thus, there was not a consistent response of thrombocytopenia to TMN-1 among any other subjects.

\section{Causal Criteria: Search for Alternative Explanations}

As a result of a detailed inquiry into the history of the patient with SAE, it was found that she had intermittently taken diclofenac, chlorzoxazone, and oxetacaine tablets for her shoulder pain, beginning on the 11th day of the TMN-1 medication, with the total dosage being five tablets of each prior to the SAE. In addition, the patient disclosed that, at about the 5- to 11-day period prior to the SAE, she had occasionally consumed Moringa oleifera tea. The tea was usually prepared by brewing about $5 \mathrm{~g}$ of $M$. oleifera tea leaves in $500 \mathrm{~cm}^{3}$ of hot water, of which she drank about $100-200 \mathrm{~cm}^{3}$. However, there were no similar symptoms among any of her family members who drank more of this beverage than she did.

Most importantly, we discovered that 4 days prior to the occurrence of the SAE, the patient had been taking continuous prescribed doses of acetaminophen, mefenamic, and chlorpheniramine for a sore throat condition. Given that all of these drugs had once been reported to have caused, or to have been associated with, thrombocytopenia, ${ }^{19-29}$ they were immediately suspected as the major alternative hypotheses.

Drug-induced thrombocytopenia is, however, a rare untoward medical occurrence, ${ }^{19}$ which is difficult to verify because of the diverse clinical pictures produced by various specific immunological platelet interactions. ${ }^{30}$ Clearly, therefore, the most important measure in determining the causal agent-the prompt withdrawal of the suspected drug $^{24}$ - does not seem feasible when multiple drugs (drug-drug and drug-herb interactions) are being used concomitantly.

\section{Discussion}

It is our view that when criteria are used in causal inference for the purpose of making herbal safety recommendations, no single criteria (of these five: consistency, strength of association, dose response, biologic plausibility, and temporality) is absolutely critical or absolutely irrelevant. We therefore conclude that, in the vast majority of situations, the criteria of causality assessment summarized in this report may be a feasible process for careful evaluation of adverse drug reactions related to all herbal medicine, namely, with appropriate temporality as the necessary criterion, followed by consistency of association and ruling out all alternative explanations.

Our establishment of an active safety system, within which we adopted a prospective observational method of monitoring subjects' untoward reactions, was aimed at providing a supplement for many of the drawbacks noted above. We believe that it is quite feasible for the detection of symptoms or signs for most, if not every, adverse event to be achieved as a result of the close cooperation between investigators trained in both conventional and Chinese medicine, along with the periodic monitoring of patients.

Taking this event as an example, the subject herself did not consider the gingival bleeding as abnormal, and regarded the petechiae and ecchymoses as ordinary signs of contusion injury. She even showed some signs of hesitation toward her proposed admission into intensive care, despite having already been informed of her low platelet counts. Nevertheless, following our emergency management, the patient is now fully aware that she may be susceptible to acetaminophen, mefenamic, or chlorpheniramine, and she is also aware that she must be careful to avoid these medications for the rest of her life.

Within our safety system, the active surveillance of the patient's platelet counts provided us with an opportunity to exclude the possibility that herbs alone were the cause of this SAE; we found that this was in fact related to the subject's comedication with conventional drugs. Such a combined approach to the consensual assessment of causality of this event would appear to have made the conclusion of this case much more objective and reliable.

\section{Acknowledgments}

This SAE occurred during the study supported by a grant from the National Science and Technology Program Office (NSTP)/Biotechnology and Pharmaceuticals (92 A322) (DOH-93-TD-I-111-004), Taiwan. We are most grateful to the 
staff for their hard work and contribution to the management and causality assessment of this event.

\section{Disclosure Statement}

No competing financial interests exist.

\section{References}

1. Cheng KF, Leung KS, Leung PC. Interactions between modern and Chinese medicinal drugs: A general review. Am J Chin Med 2003;31:163-169.

2. Izzo AA, Ernst E. Interactions between herbal medicines and prescribed drugs: A systematic review. Drugs 2001;61:21632175.

3. Abebe W. Herbal medications: Potential for adverse interactions with analgesic drugs. J Clin Pharm 2002;27:391-401.

4. Williamson EM. Drug interactions between herbal and prescription medicines. Drug Saf 2003;26:1075-1092.

5. Peng CC, Glassman PA, Trilli LE, et al. Incidence and severity of potential drug-dietary supplement interactions in primary care patients: An exploratory study of two outpatient practices. Arch Intern Med 2004;164:630-636.

6. Coxeter PD, McLachlan AJ, Duke CC, Roufogalis BD. Herbdrug interactions: An evidence-based approach. Curr Med Chem 2004;11:1513-1525.

7. Chang LC, Huang N, Chou YJ, et al. Utilization patterns of Chinese medicine and Western medicine under the National Health Insurance Program in Taiwan, a population-based study from 1997 to 2003. BMC Health Services Res 2008;8:170.

8. Joos S, Musselmann B, Szecsenyi J. Integration of complementary and alternative medicine into family practices in Germany: Results of a national survey. eCAM 2009;10:1093.

9. Barnes J. Quality, efficacy and safety of complementary medicines: Fashions, facts and the future. Part II: Efficacy and safety. Br J Clin Pharmacol 2003;55:331-340.

10. Macedo AF, Marques FB, Ribeiro CF. Can decisional algorithms replace global introspection in the individual causality assessment of spontaneously reported ADRs? Drug Saf 2006;29:697-702.

11. Lai JN, Hwang JS, Chen HJ, Wang JD. Finished herbal product as an alternative treatment for menopausal symptoms in climacteric women. J Altern Complement Med 2005;11:1075-1084.

12. Evans A. Causation and disease: A chronological journey. Am J Epidemiol 1978;108:249-258.

13. Yerushalmy J, Palmer CE. On the methodology of investigations of etiologic factors in chronic diseases. $\underline{\mathrm{J} \text { Chron Dis }}$ 1959;10:27-40.

14. Susser M. Judgment and causal inference: Criteria in epidemiologic studies. Am J Epidemiol 1977;105:1-15.

15. Hill AB. The environment and disease: Association or causation? Proc Roy Soc Med 1965;58:295-300.
16. Wang JD. Causal inference and decisions. In: Basic Principles and Practical Applications in Epidemiological Research. Singapore: World Scientific, 2002:57-79.

17. Rothman KJ, Greenland S, Poole C, Lash TL. Causation and causal inference. In: Rothman KJ, Greenland S, Lash TL, eds. Modern Epidemiology. 3rd ed. Philadelphia: Wolters Kluwer Health/Lippincott Williams \& Wilkins, 2008;5-31.

18. Page RL, Lawrence JD. Potentiation of warfarin by dong quai. Pharmacotherapy 1999;19:870-876.

19. Kaufman DW, Kelly JP, Johannes CB, et al. Acute thrombocytopenic purpura in relation to the use of drugs. Blood 1993;82:2714-2718.

20. Pedersen-Bjergaard U, Andersen M, Hansen PB. Drugspecific characteristics of thrombocytopenia caused by noncytotoxic drugs. Eur J Clin Pharmacol 1998;54:701-706.

21. Pedersen-Bjergaard U, Andersen M, Hansen PB. Druginduced thrombocytopenia: Clinical data on 309 cases and the effects of corticosteroid therapy. Eur J Clin Pharmacol 1997;52:183-189.

22. George JN, Raskob GE, Shah SR, et al. Drug-induced thrombocytopenia: A systematic review of published case reports. Ann Intern Med 1998;129:886-890.

23. Pedersen Bjergaard U, Andersen M, Hansen PB. Thrombocytopenia induced by non-cytotoxic drugs in Denmark, 1968-1991. J Intern Med 1996;239:509-515.

24. Heading RC. Purpura and paracetamol. BMJ 1968;3:743-744.

25. Eisner EV, Shahidi NT. Immune thrombocytopenia due to a drug metabolite. NEJM 1972;287:376-381.

26. Kornberg A, Polliack A. Paracetamol-induced thrombocytopenia and haemolytic anaemia. Lancet 1978;2:1159.

27. Scheinberg IH. Thrombocytopenic reaction to aspirin and acetaminophen. NEJM 1979;300:678.

28. Shoenfeld Y, Shaklai M, Livni E, Pinkhas J. Thrombocytopenia from acetaminophen. NEJM 1980;303:47.

29. Murphy WG, Kelton JG. Idiosyncratic drug-induced thrombocytopenia. Curr Stud Hematol Blood Transfus 1988; 54:71-88.

30. Barnes J, Mills SY, Abbot NC, et al. Different standards for reporting ADRs to herbal remedies and conventional OTC medicines: Face-to-face interviews with 515 users of herbal remedies. Br J Clin Pharmacol 1998;45:496-500.

Address correspondence to: Jung-Der Wang, MD, ScD

Department of Public Health

National Cheng Kung University No. 1, University Road

Tainan 107

Taiwan

E-mail: jdwang121@gmail.com 